UMN-TH-1763/99

TPI-MINN-99/27

gr-qc/9905032

May 1999

\title{
Gödel-type Universes in String-inspired Charged Gravity
}

\author{
P. $\operatorname{Kanti}^{(a)}$ and C.E. Vayonakis ${ }^{(b)}$ \\ (a) Theoretical Physics Institute, School of Physics and Astronomy, \\ University of Minnesota, Minneapolis, MN 55455, USA \\ (b) Division of Theoretical Physics, Physics Department, \\ University of Ioannina, Ioannina GR-451 10, Greece
}

\begin{abstract}
We consider a string-inspired, gravitational theory of scalar and electromagnetic fields and we investigate the existence of axially-symmetric, Gödel-type cosmological solutions. The neutral case is studied first and an "extreme" Gödel-type rotating solution, that respects the causality, is determined. The charged case is considered next and two new configurations for the, minimally-coupled to gravity, electromagnetic field are presented. Another configuration motivated by the expected distribution of currents and charges in a rotating universe is studied and shown to lead to a Gödel-type solution for a space-dependent coupling function. Finally, we investigate the existence of Gödel-type cosmological solutions in the framework of the one-loop corrected superstring effective action and we determine the sole configuration of the electromagnetic field that leads to such a solution. It turns out that, in all the charged cases considered, Closed Timelike Curves do appear and the causality is always violated.
\end{abstract}

Emails: yiota@physics.umn.edu, cevayona@cc.uoi.gr 


\section{Introduction}

Einstein's Theory of Relativity has been exhaustively studied for many decades and a plethora of solutions, both mathematically and physically interesting, have been determined. One of the most fascinating cosmological solutions that emerged from Einstein's equations was the Gödel-type rotating universe [1]. The feature that was most appealing and intensely attracted the interest of scientists to these cosmological models was the fact that they admit Closed Timelike Curves. This leads to the violation of causality in the universe [2] and to the possibility of time-travel into our past or future [3].

Since their discovery in 1949, several versions and modifications of the Gödel cosmological solutions have been considered. Apart from the perfect fluid that gave rise to the original Gödel solutions, extra fields, i.e. vector [4] [5] [6], scalar [7], spinor [8] (and their combinations [9] [10] [11]) and even tachyon fields [12], were gradually introduced. Higher-derivative gravitational terms were also considered in an attempt to introduce quantum corrections to Einstein's theory of gravity [13]. Recently, geometrical aspects of Gödel-type solutions have also been studied, either determining solutions that could be interpreted as the exterior of Gödel spacetimes [14 or connecting the 3+1 Gödel geometry with a 2+1 anti-de Sitter subspace [15]. Five-dimensional models that admit generalized Gödel-type solutions have also been constructed [16] and the same type of solutions was proved to arise also in Riemann-Cartan spacetimes with non-zero torsion [17].

As it is well known, superstring theory is the most plausible theory nowadays for the unification of all forces and the formulation of a quantum theory of gravity. The effective theory of heterotic superstrings at low energies provides us with a gravitational theory from which the Einstein's theory of gravitation automatically emerges when one ignores all higherloop stringy corrections and assigns constant values to the scalar fields of the theory. On the other hand, when one takes into account these extra terms, a generalized theory of gravity arises which leads to important modifications when compared to the Theory of General Relativity. The question of what happens to the causal pathologies of the Gödeltype cosmological solutions when one introduces stringy corrections to Einstein's theory of gravity inevitably arises. It is, then, necessary to investigate the existence of Gödeltype solutions and the appearance or not of Closed Timelike Curves in the framework of the superstring effective theory. Barrow and Dabrowski [18] tried to answer this question and studied the one-loop corrected superstring effective theory in the string frame (and the lowest order in the Einstein frame) by considering a non-zero dilaton and axion field. They found that, in the context of this specific theory, Gödel universes do not contain Closed Timelike Curves and the causality need not be violated. Here, we will try to extend their work and study the full one-loop superstring effective theory in the Einstein frame. We will consider all four scalar fields, as well as higher-derivative gravitational and electromagnetic terms. However, our analysis will be greatly simplified by the fact that all higher-derivative gravitational terms identically vanish for a Gödel spacetime.

The structure of this article is as follows : in section 2, we present the action functional 
of the theory and derive the corresponding equations of motion in an axially-symmetric spacetime background. In section 3, we study the neutral case where scalar fields minimallycoupled to gravity give rise to Gödel-type cosmological solutions. The charged case is considered in section 4 and the equations of motion are conveniently formulated. In the same section, we deal with the problem of the simultaneous existence of an electromagnetic and scalar fields all minimally-coupled to gravity. All the possible configurations for the electromagnetic components that lead to Gödel-type rotating universes are determined and the solution for the scalar fields is presented. In section 5, the physically favoured case of a constant magnetic field along the rotation axis together with a radial electric and magnetic component is studied and proved to be compatible with an axially-symmetric universe only in the case of a space-dependent coupling function. The non-trivial interaction between the electromagnetic and the scalar fields in the framework of the one-loop corrected, superstring, effective theory is the basic feature of the gravitational theory considered in section 6 . There, the possible configurations of the electromagnetic field that lead to Gödel-type universes are re-examined and the solution for the scalar fields is determined. Section 7 is devoted to the conclusions derived from our analysis while some useful formulae and equations are displayed in the Appendix at the end of the paper.

\section{Action Functional and Equations of Motion of the Theory}

We consider the following, string-inspired, generalized theory of gravity which describes the coupling of four scalar fields and the electromagnetic field to gravity

$$
\begin{aligned}
S_{e f f} & =\int d^{4} x \sqrt{-g}\left\{\frac{R}{2}+\frac{1}{4}\left(\partial_{\mu} \phi\right)^{2}+\frac{1}{4} e^{-2 \phi}\left(\partial_{\mu} a\right)^{2}+\frac{3}{4}\left(\partial_{\mu} \sigma\right)^{2}\right. \\
& \left.+\frac{3}{4} e^{-2 \sigma}\left(\partial_{\mu} b\right)^{2}+f_{1} \mathcal{R}_{G B}^{2}+f_{2} \mathcal{R} \tilde{\mathcal{R}}+f_{3} F^{\mu \nu} F_{\mu \nu}+f_{4} F \tilde{F}\right\} .
\end{aligned}
$$

In the above, $\phi$ is the dilaton, $a$ and $b$ the axions and $\sigma$ the modulus field. The gravitational and electromagnetic quantities that appear in the above expression are defined as

$$
\begin{aligned}
\mathcal{R}_{G B}^{2} & \equiv R_{\mu \nu \kappa \lambda} R^{\mu \nu \kappa \lambda}-4 R_{\mu \nu} R^{\mu \nu}+R^{2} \\
\mathcal{R} \tilde{\mathcal{R}} & \equiv \eta^{\mu \nu \rho \sigma} R_{\mu \nu}^{\kappa \lambda} R_{\rho \sigma \kappa \lambda} \\
F \tilde{F} & \equiv \eta^{\mu \nu \rho \sigma} F_{\mu \nu} F_{\rho \sigma} .
\end{aligned}
$$

In the framework of the one-loop corrected, effective theory of heterotic superstrings at 
low energies the coupling functions $f_{i}$ have the form

$$
\begin{array}{cl}
f_{1} \equiv \alpha^{\prime}\left(\frac{e^{\phi}}{8 g^{2}}+\Delta\right) & f_{2} \equiv \alpha^{\prime}\left(\frac{a}{8 g^{2}}+\Theta\right), \\
f_{3} \equiv \alpha^{\prime}\left(-\frac{e^{\phi}}{8 g^{2}}+\hat{\Delta}\right) \quad, \quad f_{4} \equiv \alpha^{\prime}\left(-\frac{a}{8 g^{2}}+\hat{\Theta}\right),
\end{array}
$$

where $\alpha^{\prime}$ is the inverse string tension and the functions $\Delta, \Theta, \hat{\Delta}$ and $\hat{\Theta}$ depend only on the scalar fields $b$ and $\sigma$. However, during the following sections, we are going to assume that the coupling functions $f_{i}$ are zero, constant, space-dependent or field-dependent according to the type of gravitational theory considered each time.

We assume that the universe is described by the following axially-symmetric line-element

$$
\begin{aligned}
d s^{2} & =[d t+C(r) d \varphi]^{2}-d r^{2}-d z^{2}-D^{2}(r) d \varphi^{2} \\
& =d t^{2}-d r^{2}-d z^{2}-\left(D^{2}-C^{2}\right) d \varphi^{2}+2 C(r) d t d \varphi .
\end{aligned}
$$

By making use of the non-vanishing components of the curvature tensor $R_{\mu \nu \rho \sigma}$, of the Ricci tensor $R_{\mu \nu}$ and the scalar curvature $R$, which are given in the Appendix, it is easy to see that both of the higher-derivative gravitational terms $\mathcal{R}_{G B}^{2}$ and $\mathcal{R} \tilde{\mathcal{R}}$ vanish identically. Then, the variation of the action functional of the theory with respect to the scalar fields, the metric tensor and the electromagnetic potential leads to the following set of equations of motion

$$
\begin{gathered}
\frac{1}{\sqrt{-g}} \partial_{\mu}\left[\sqrt{-g} \partial^{\mu} \phi\right]=-e^{-2 \phi}\left(\partial_{\mu} a\right)^{2}+2 \frac{\partial f_{3}}{\partial \phi} F^{\mu \nu} F_{\mu \nu}, \\
\frac{1}{\sqrt{-g}} \partial_{\mu}\left[\sqrt{-g} e^{-2 \phi} \partial^{\mu} a\right]=2 \frac{\partial f_{4}}{\partial a} F \tilde{F} \\
\frac{1}{\sqrt{-g}} \partial_{\mu}\left[\sqrt{-g} \partial^{\mu} \sigma\right]=-e^{-2 \sigma}\left(\partial_{\mu} b\right)^{2}+\frac{2}{3} \frac{\partial f_{3}}{\partial \sigma} F^{\mu \nu} F_{\mu \nu}+\frac{2}{3} \frac{\partial f_{4}}{\partial \sigma} F \tilde{F}, \\
\frac{1}{\sqrt{-g}} \partial_{\mu}\left[\sqrt{-g} e^{-2 \sigma} \partial^{\mu} b\right]=\frac{2}{3} \frac{\partial f_{3}}{\partial b} F^{\mu \nu} F_{\mu \nu}+\frac{2}{3} \frac{\partial f_{4}}{\partial b} F \tilde{F} \\
R_{\mu \nu}-\frac{1}{2} g_{\mu \nu} R+4 f_{3}\left(F_{\mu}{ }^{\sigma} F_{\nu \sigma}-\frac{1}{4} g_{\mu \nu} F^{\rho \sigma} F_{\rho \sigma}\right)=-\frac{1}{2}\left(\partial_{\mu} \phi\right)\left(\partial_{\nu} \phi\right) \\
+\frac{1}{4} g_{\mu \nu}\left(\partial_{\rho} \phi\right)^{2}-\frac{e^{-2 \phi}}{2}\left(\partial_{\mu} a\right)\left(\partial_{\nu} a\right)+\frac{1}{4} g_{\mu \nu} e^{-2 \phi}\left(\partial_{\rho} a\right)^{2}-\frac{3}{2}\left(\partial_{\mu} \sigma\right)\left(\partial_{\nu} \sigma\right) \\
+\frac{3}{4} g_{\mu \nu}\left(\partial_{\rho} \sigma\right)^{2}-\frac{3}{2} e^{-2 \sigma}\left(\partial_{\mu} b\right)\left(\partial_{\nu} b\right)+\frac{3}{4} e^{-2 \sigma} g_{\mu \nu}\left(\partial_{\rho} b\right)^{2}
\end{gathered}
$$




$$
\frac{1}{\sqrt{-g}} \partial_{\mu}\left[\sqrt{-g} f_{3} F^{\mu \nu}\right]+\frac{1}{\sqrt{-g}} \partial_{\mu}\left[\sqrt{-g} f_{4} \tilde{F}^{\mu \nu}\right]=0,
$$

where we have assumed that the functions $f_{3}$ and $f_{4}$ depend on spacetime either directly of through their dependence on the scalar fields. In the latter case, there is a non-trivial coupling between the scalar fields of the theory and the electromagnetic field.

\section{The Neutral Case}

In this section, we ignore the electromagnetic field and concentrate our attention on the Einstein's equations, $G_{\mu \nu}=-T_{\mu \nu}^{s}$ where $T_{\mu \nu}^{s}$ is the energy-momentum tensor of the scalar fields. The non-vanishing components of both the Einstein and the energy-momentum tensor are also given in the Appendix. We notice that simple relations hold between the various components of $T_{\mu \nu}^{s}$, namely

$$
T_{z z}^{s}=-T_{r r}^{s} \quad, \quad T_{\varphi \varphi}^{s}=\left(C^{2}-D^{2}\right) T_{t t}^{s} \quad, \quad T_{t \varphi}^{s}=C T_{t t}^{s} .
$$

By making use of the above relations, we can determine the solution for the metric functions $C$ and $D$ in the presence of multiple scalar fields in the theory. Rearranging the $(t t)$ and $(t \varphi)$ components of the Einstein's equation as well as the $(t t)$ and $(\varphi \varphi)$ components, we find the constraints

$$
C^{\prime \prime}=\frac{C^{\prime} D^{\prime}}{D} \quad, \quad C^{2}=D D^{\prime \prime},
$$

respectively, where the prime denotes derivative with respect to the radial coordinate $r$. However, doing the same with the $(r r)$ and $(z z)$ components, we are led to the result $D^{\prime \prime}=0$. Then, the general solution of the above system is : $D(r)=d_{1} r+d_{2}$ and $C(r)=c_{1}$. If the line-element (2.6) is to describe a rotating universe with the function $C(r)$ being the analog of its angular momentum, then on the $z$-axis, that is in the limit $r \rightarrow 0$, both of the functions $C(r)$ and $D^{2}-C^{2}$ must vanish. This leads to $c_{1}=d_{2}=0$ and the vanishing of the $g_{t \varphi}$ component of the metric tensor. Redefining the angular coordinate $\varphi$ in order to absorb the arbitrary constant $d_{1}$, the line-element (2.6) reduces to that of a non-rotating, sphericallysymmetric universe. Finally, by rearranging the $(t t)$ and $(r r)$ components of the Einstein's equations, we obtain the following relations

$$
\begin{aligned}
& \left(\partial_{r} \phi\right)^{2}+e^{-2 \phi}\left(\partial_{r} a\right)^{2}+3\left(\partial_{r} \sigma\right)^{2}+3 e^{-2 \sigma}\left(\partial_{r} b\right)^{2}=0, \\
& \left(\partial_{z} \phi\right)^{2}+e^{-2 \phi}\left(\partial_{z} a\right)^{2}+3\left(\partial_{z} \sigma\right)^{2}+3 e^{-2 \sigma}\left(\partial_{z} b\right)^{2}=0 .
\end{aligned}
$$

According to the above constraints, each one of the derivatives of the scalar fields with respect to $r$ or $z$ must vanish. In other words, the only acceptable solution for the scalar

fields is the trivial one : $\phi=\phi_{0}, a=a_{0}, \sigma=\sigma_{0}$ and $b=b_{0}$ where $\phi_{0}, a_{0}, \sigma_{0}$ and $b_{0}$ are arbitrary constants. 
However, if we include in the theory the cosmological constant $\Lambda$, the Einstein tensor $G_{\mu \nu}$ should be replaced by $G_{\mu \nu}+\Lambda g_{\mu \nu}$. In this case, the constraints (3.2) are still valid but, now, the rearrangement of the $(r r)$ and $(z z)$ components of the Einstein's equations gives the result

$$
\frac{D^{\prime \prime}}{D}=-2 \Lambda
$$

The combination of the above relation with the second one of (3.2) leads to : $C^{\prime 2}=-2 \Lambda D^{2}$ which means that the cosmological constant must be always negative in order to ensure the reality of the functions $C$ and $D$. If we demand that, in the limit $r \rightarrow 0$, both of the functions $C$ and $D^{2}-C^{2}$ must vanish, the general solution of the system (3.2)-(3.5) takes the form

$$
\begin{aligned}
& D(r)=\frac{1}{m} \sinh (m r), \\
& C(r)=\frac{1}{m}[\cosh (m r)-1]=\frac{2}{m} \sinh ^{2}\left(\frac{m r}{2}\right),
\end{aligned}
$$

where $m^{2}=-2 \Lambda$. The above solution for the spacetime is a Gödel-type solution which is defined as [1] [10]

$$
d s^{2}=\left[d t+\frac{4 \Omega}{m^{2}} \sinh ^{2}\left(\frac{m r}{2}\right) d \varphi\right]^{2}-d r^{2}-d z^{2}-\frac{\sinh ^{2}(m r)}{m^{2}} d \varphi^{2},
$$

where again $m^{2}=-2 \Lambda$ and $\Omega$ is the rate of the rigid rotation of the matter around the $z$-axis. In the case of the original Gödel universe, the matter is a perfect-fluid with an energy-momentum tensor of the form $T_{\mu \nu}=\rho V_{\mu} V_{\nu}$, where $V^{\mu}=\delta_{0}^{\mu}$ the fluid four-vector velocity and $\rho$ the density of matter. The $g_{\varphi \varphi}$ component of the Gödel metric (3.8) has the form

$$
g_{\varphi \varphi}=-\frac{4}{m^{2}} \sinh ^{2}\left(\frac{m r}{2}\right)\left[1+\left(1-\frac{4 \Omega^{2}}{m^{2}}\right) \sinh ^{2}\left(\frac{m r}{2}\right)\right]
$$

and it is obvious that for $4 \Omega^{2}>m^{2}$, the $g_{\varphi \varphi}$ component may become positive for a range of values of the radial coordinate $r$ leading to the existence of Closed Timelike Curves (CTC's). The solution found above (3.6)-(3.7) constitutes a Gödel-type solution with $4 \Omega^{2}=m^{2}$. It is, actually, a limiting case of a Gödel-type universe that ensures the non-existence of Closed Timelike Curves. The same type of solution was found in the case of an electromagnetic field minimally coupled to gravity [10] and in the framework of a higher-derivative gravitational theory [13] and it was shown that this "extreme" Gödel-type solution, although "on the verge" of displaying the breakdown of causality, is free of any Closed Timelike Curves [13] [19].

Next, we concentrate our attention on the scalar fields. Their dependence on the spatial coordinates is strongly restricted by the constraint

$$
\partial_{r} \phi \partial_{z} \phi+e^{-2 \phi} \partial_{r} a \partial_{z} a+3 \partial_{r} \sigma \partial_{z} \sigma+3 e^{-2 \sigma} \partial_{r} b \partial_{z} b=0
$$

which follows from the $(r z)$ component of the Einstein's equations. We shall consider the most general case in which all the scalar fields depend on the $r$ and $z$ coordinates due to the 
staticity and axial symmetry of the spacetime. The remaining components of the Einstein's equations give the following constraints

$$
\begin{aligned}
-2 \Lambda & =\left(\partial_{r} \phi\right)^{2}+\left(\partial_{z} \phi\right)^{2}+e^{-2 \phi}\left[\left(\partial_{r} a\right)^{2}+\left(\partial_{z} a\right)^{2}\right]+3\left[\left(\partial_{r} \sigma\right)^{2}+\left(\partial_{z} \sigma\right)^{2}\right] \\
& +3 e^{-2 \sigma}\left[\left(\partial_{r} b\right)^{2}+\left(\partial_{z} b\right)^{2}\right] \\
-2 \Lambda & =-\left(\partial_{r} \phi\right)^{2}+\left(\partial_{z} \phi\right)^{2}+e^{-2 \phi}\left[-\left(\partial_{r} a\right)^{2}+\left(\partial_{z} a\right)^{2}\right]+3\left[-\left(\partial_{r} \sigma\right)^{2}+\left(\partial_{z} \sigma\right)^{2}\right] \\
& +3 e^{-2 \sigma}\left[-\left(\partial_{r} b\right)^{2}+\left(\partial_{z} b\right)^{2}\right] .
\end{aligned}
$$

Rearranging the above expressions, we are led to the results

$$
\begin{aligned}
& \left(\partial_{r} \phi\right)^{2}+e^{-2 \phi}\left(\partial_{r} a\right)^{2}+3\left(\partial_{r} \sigma\right)^{2}+3 e^{-2 \sigma}\left(\partial_{r} b\right)^{2}=0 \\
& \left(\partial_{z} \phi\right)^{2}+e^{-2 \phi}\left(\partial_{z} a\right)^{2}+3\left(\partial_{z} \sigma\right)^{2}+3 e^{-2 \sigma}\left(\partial_{z} b\right)^{2}=-2 \Lambda .
\end{aligned}
$$

The former constraint is satisfied only if we assume that the scalar fields do not depend on the radial coordinate. In this case, the supplementary constraint (3.10) is automatically satisfied. The latter constraint leads to the following dependence of the scalar fields on the coordinate $z$

$$
\begin{array}{ll}
\phi=\phi_{0}+\phi_{1} z & a=a_{0}+a_{1} e^{\phi}, \\
\sigma=\sigma_{0}+\sigma_{1} z & ,
\end{array}
$$

where $\phi_{i}, a_{i}, \sigma_{i}$ and $b_{i}$ are constants. If we substitute the above expressions for the scalar fields in their equations of motion, we find some further constraints, namely $\phi_{1} a_{1}=0$ and $\sigma_{1} b_{1}=0$, which means that not both of the pairs $(\phi, \sigma)$ and $(a, b)$ can have a non-trivial solution. If we choose to set $\phi_{1}=\sigma_{1}=0$ in order to satisfy the above constraints, then, the second pair of scalar fields $(a, b)$ is inevitably led to the trivial solution as well. If, on the other hand, we set $a_{1}=b_{1}=0$, the dilaton and modulus field can still preserve a non-trivial form. As a result, the most interesting solution for the scalar fields is the following

$$
\phi=\phi_{0}+\phi_{1} z \quad, \quad a=a_{0} \quad, \quad \sigma=\sigma_{0}+\sigma_{1} z \quad, \quad b=b_{0},
$$

where

$$
-2 \Lambda=\phi_{1}^{2}+3 \sigma_{1}^{2}
$$

Similar results for the dilaton and axion field $a$ were found by Barrow and Dabrowski 18 . Here, we have also included the axion field $b$ and the modulus $\sigma$, that are present in the lowenergy effective string theory, and shown that the solution for the metric remains unchanged. Moreover, from the above analysis, it is easy to conclude that a theory with an arbitrary number of multiple scalar fields formulated in the following way

$$
S=\int d^{4} x \sqrt{-g}\left\{\frac{R}{2}-\Lambda+\sum_{i=1}^{N}\left(\frac{1}{4} \partial_{\mu} \phi_{i} \partial^{\mu} \phi_{i}+\frac{1}{4} e^{-2 \phi_{i}} \partial_{\mu} a_{i} \partial^{\mu} a_{i}\right)\right\}
$$


always accepts axially-symmetric spacetime backgrounds of the form (2.6) and (3.6)-(3.7) with the scalar fields given by

$$
\phi_{i}=\phi_{0}^{i}+\phi_{1}^{i} z \quad, \quad a=a_{0}^{i}
$$

and with the constants $\phi_{1}^{i}$ satisfying the constraint

$$
-2 \Lambda=\sum_{i=1}^{N}\left(\phi_{1}^{i}\right)^{2} .
$$

\section{$4 \quad$ EM and Scalar Fields : Minimal Coupling}

Next, we assume that an electromagnetic field, in the form of $F_{\mu \nu} F^{\mu \nu}$ and $F \tilde{F}$, is included in the theory with coupling functions $f_{3}$ and $f_{4}$, respectively. In this case, the Einstein's equations are supplemented by Maxwell's equations that come from the equation of motion of the electromagnetic field (2.12). The exact form of both sets of equations are displayed

in the Appendix. The relations (3.1) between the components of $T_{\mu \nu}^{s}$ still hold and may be used once again in order to derive a number of constraints on the metric functions $C$ and $D$. Rearranging accordingly eqs. A.17)-(A.18), (A.16)-(A.19) and (A.16)-(A.20), we are led to the following constraints

$$
\begin{gathered}
\frac{D^{\prime \prime}}{D}+2 \Lambda+4 f_{3}\left[F_{r z}^{2}+F_{t \varphi}^{2}\left(g^{t \varphi} g^{t \varphi}-g^{t t} g^{\varphi \varphi}\right)\right]=0 \\
C C^{\prime \prime}-D D^{\prime \prime}+C^{\prime 2}-\frac{C C^{\prime} D^{\prime}}{D}+4 f_{3}\left[F_{\varphi r}^{2}+F_{\varphi z}^{2}-\left(C^{2}-D^{2}\right)\left(F_{t r}^{2}+F_{t z}^{2}\right)\right]=0, \\
\frac{C^{\prime \prime}}{2}-\frac{C^{\prime} D^{\prime}}{2 D}+4 f_{3}\left[F_{t r} F_{\varphi r}+F_{t z} F_{\varphi z}-C\left(F_{t r}^{2}+F_{t z}^{2}\right)\right]=0 .
\end{gathered}
$$

Moreover, one could express the derivatives of the scalar fields with respect only to $r$ or only to $z$ in terms of the metric functions and the various components of the electromagnetic field. Adding or subtracting the $(t t)$ and $(r r)$ components of the Einstein's equations and by making use of the above constraints (4.1)-(4.3), we obtain the results

$$
\begin{aligned}
\left(\partial_{r} \phi\right)^{2}+e^{-2 \phi}\left(\partial_{r} a\right)^{2}+3\left(\partial_{r} \sigma\right)^{2}+ & 3 e^{-2 \sigma}\left(\partial_{r} b\right)^{2}=8 f_{3}\left(F_{r z}^{2}-F_{t r}^{2}\right) \\
+ & \frac{8 f_{3}}{D^{2}}\left(F_{\varphi t}^{2}-F_{\varphi z}^{2}-C^{2} F_{t z}^{2}+2 C F_{t z} F_{\varphi z}\right) \\
\left(\partial_{z} \phi\right)^{2}+e^{-2 \phi}\left(\partial_{z} a\right)^{2}+3\left(\partial_{z} \sigma\right)^{2}+ & 3 e^{-2 \sigma}\left(\partial_{z} b\right)^{2}=\frac{C^{\prime 2}}{D^{2}}+8 f_{3}\left(F_{r z}^{2}+F_{t r}^{2}\right) \\
& +\frac{8 f_{3}}{D^{2}}\left(F_{\varphi t}^{2}+F_{\varphi z}^{2}+C^{2} F_{t z}^{2}-2 C F_{t z} F_{\varphi z}\right)
\end{aligned}
$$


The substitution of eqs. (A.16)-(A.20) by the constraints 4.1)-(4.5) allows us to determine first the form of the metric functions $C$ and $D$ in terms only of the non-vanishing components of the electromagnetic field, through eqs. (4.1)-(4.3), and subsequently the solution for the scalar fields, through eqs. (4.4)-(4.5).

We start the study of the charged case by first considering the minimal coupling of the electromagnetic and scalar fields to the gravitational field. As a result, we are going to assume that both of the coupling functions $f_{3}$ and $f_{4}$ do not depend on spacetime and set $f_{3}=f_{4}=-1 / 4$. The necessary and sufficient conditions for the existence and spacetime homogeneity, at the same time, of a Gödel-type axially-symmetric universe are the following [10

$$
\frac{D^{\prime \prime}}{D}=\text { const. }=-2 \Lambda=m^{2} \quad, \quad \frac{C^{\prime}}{D}=\text { const. }=2 \Omega
$$

By studying eq. (4.1), it is straightforward to see that the first condition is satisfied if we assume that $F_{r z}=F_{t \varphi}=0$, i.e. if that both the electric and magnetic components along the $\hat{\varphi}$ direction vanish, an assumption easily accepted given the cylindrical symmetry of the problem. The second condition is satisfied if we assume that the combination of the components of the electromagnetic field that appears inside the brackets in eq. (4.3) vanishes, too. Then, eq. (4.2) provides us with a relation between the different parameters of the theory. Note, that once these two conditions (4.6) are satisfied, a Gödel-type cosmological solution appears independently of the existence or not of scalar fields in our theory.

Gödel-type cosmological solutions, in the case of a minimally coupled electromagnetic field but in the absence of scalar fields, already exist in the literature : the Som-Raychaudhuri solution [4] and the Rebouças solution [5]. The Som-Raychaudhuri ansatz for the electromagnetic field has the form

$$
F^{\varphi r}(r, z)=-\frac{\sqrt{4 \Omega^{2}-m^{2}}}{D(r)} \quad, \quad F^{r t}(r, z)=C(r) F^{\varphi r} .
$$

When written in terms of covariant components, the above ansatz gives rise only to a magnetic field along the $z$ axis : $B_{z}=F_{\varphi r}=\sqrt{4 \Omega^{2}-m^{2}} D(r)$. In this case, both of the conditions (4.6) are satisfied giving rise to a Gödel-type solution for the spacetime while the equations for the electromagnetic field (A.27)-(A.30) are satisfied only if we assume the presence of a continuous electric-charge distribution with charge density given by : $\rho=\frac{\Omega}{2} \sqrt{4 \Omega^{2}-2 m^{2}}$. On the other hand, the Rebouças solution assumes the ansatz

$$
F_{t z}=A_{0} \sin (2 \Omega z) \quad, \quad F_{\varphi z}=C(r) F_{t z} \quad, \quad F_{\varphi r}=-A_{0} D(r) \cos (2 \Omega z),
$$

where $A_{0}=\sqrt{4 \Omega^{2}-m^{2}}$. The above ansatz also satisfies the conditions (4.6) as well as the source-free Maxwell equations (A.27)-(A.30).

As Raychaudhuri and Guha Thakurta have pointed out in their work [6], there are more than one ansatzes for the form of the electromagnetic field that may lead to the same Gödeltype cosmological solution. In this section, we make a thorough analysis of the possible 
configurations of the electromagnetic field and present the remaining solutions. Since the first of the conditions (4.6) is easily satisfied by setting $F_{r z}=F_{t \varphi}=0$, we concentrate our attention on the constraints (4.2)-(4.3) looking for all the possible, non-trivial combinations of the components of the electromagnetic field that respect the second condition. We notice that these constraints are characterized by a "duality", i.e. they remain invariant under the interchange of the indices $r$ and $z$ in the components of the electromagnetic field. As a result, we are led to consider the "dual" forms of the aforementioned solutions (4.7) and (4.8). We start with the Som-Raychaudhuri-dual solution and consider the ansatz

$$
B_{r}=F_{z \varphi}=\sqrt{4 \Omega^{2}-m^{2}} D(r) .
$$

It is easy to see that this ansatz satisfies both of the constraints (4.6) leading to a Gödel-type solution of the form (3.8). As in the original Som-Raychaudhuri solution, we are forced to introduce a source term in order to satisfy Maxwell's equations (A.27)-(A.30). However, in this case the source term corresponds to a distribution of magnetic monopoles instead of electric. Following Dirac's example [20], we introduce, apart from the electric four-current $j^{\mu}=\{\rho, \vec{j}\}$, a magnetic four-current $k^{\mu}=\{\eta, \vec{k}\}$ and write Maxwell's equations (2.12) in the more general form

$$
\frac{1}{\sqrt{-g}} \partial_{\mu}\left[\sqrt{-g}\left(f_{3} F^{\mu \nu}+f_{4} \tilde{F}^{\mu \nu}\right)\right]=-\left(j^{\mu}+k^{\mu}\right) .
$$

Then, the substitution of the ansatz (4.9) into the above equation leads to the determination of a magnetic charge density equal to :

$$
\eta=-\frac{\sqrt{4 \Omega^{2}-m^{2}}}{4} \frac{D^{\prime}}{D} .
$$

The magnetic monopoles are spread all over the universe as their corresponding density vanishes at the origin while it approaches a constant value at infinity.

Finally, the "dual" ansatz of the Rebouças solution (4.8) can be written in the form

$$
F_{t r}=\frac{\sqrt{4 \Omega^{2}-m^{2}}}{\sqrt{2}} \quad, \quad F_{\varphi r}=C(r) F_{t r} \quad, \quad F_{\varphi z}=D(r) F_{t r} .
$$

As the original Rebouças solution, the above "dual" solution leads to a Gödel-type universe (3.8) and satisfies the source-free Maxwell's equations. As a matter of fact, the substitution of the above ansatz into the generalized Maxwell's equations (4.10) leads to non-vanishing expressions for the electric and magnetic-charge densities, i.e.

$$
\rho=-\frac{\sqrt{4 \Omega^{2}-m^{2}}}{\sqrt{2}} D^{\prime} \quad, \quad \eta=\frac{\sqrt{4 \Omega^{2}-m^{2}}}{\sqrt{2}} D^{\prime}
$$

which, however, being exactly opposite, cancel each other. If we set $F_{\varphi z}=0$, the solution for the spacetime remains unchanged and, as a result, the following combination of $F_{t r}$ and $F_{\varphi r}$ alone

$$
F_{t r}=\sqrt{4 \Omega^{2}-m^{2}} \quad, \quad F_{\varphi r}=C(r) F_{t r}
$$


is an acceptable solution of eqs. (4.1)-(4.3) by itself. However, the vanishing of the component $F_{\varphi z}$ necessitates the introduction of a source-term into Maxwell's equations. The corresponding electric-charge density that follows has the form

$$
\rho=\frac{\sqrt{4 \Omega^{2}-m^{2}}}{4} \frac{D^{\prime}}{D}
$$

As in the case of the Som-Raychaudhuri-dual solution, the electric monopoles are spread all over the universe. The "dual" solution of this last combination can be written as

$$
F_{t z}=\sqrt{4 \Omega^{2}-m^{2}} \quad, \quad F_{\varphi z}=C(r) F_{t z}
$$

and leads, as we expect, to a Gödel-type universe of the form (3.8). The electric-charge density found above is substituted, in this dual case, by a magnetic-charge density of the form $\eta=\frac{\Omega}{2} \sqrt{4 \Omega^{2}-m^{2}}$. The above density corresponds to a homogeneous, constant distribution of magnetic monopoles.

The modification (4.10) of Maxwell's equations is acceptable only in the case where the corresponding source-terms do not act as an additional source for the gravitational field and, thus, do not destroy the Gödel-type cosmological solutions. In other words, the introduction of charge and current densities in the Maxwell's equations result from the introduction of the term $-2\left(j^{\mu}+k^{\mu}\right) A_{\mu}$ in the Lagrangian of the theory, where $A_{\mu}$ is the electromagnetic potential. In principle, such a term could enter the Einstein's equations and cause the violation of the Gödel conditions (4.6). In the cases of the Som-Raychaudhuri ansatz (4.7), the Rebouças ansatz (4.8) and their "dual" ansatzes (4.9) and (4.12), the sourceterm $\left(j^{\mu}+k^{\mu}\right) A_{\mu}$ vanishes identically and, thus, does not affect the Einstein's equations and the solution for the spacetime. On the other hand, the ansatzes (4.14) and (4.16) introduce a non-vanishing source-term in the Lagrangian which depends non-trivially on the spacetime coordinates and thus modifies the Gödel-type solution. As a result, these two ansatzes fail to lead to a Gödel-type rotating universe and, thus, they will not be considered any longer.

All of the above solutions for the spacetime and electromagnetic field are compatible with the existence of scalar fields, minimally-coupled to gravity, in the theory. The constraints (4.4)-(4.5) can, now, be written as

$$
\begin{aligned}
& \left(\partial_{r} \phi\right)^{2}+e^{-2 \phi}\left(\partial_{r} a\right)^{2}+3\left(\partial_{r} \sigma\right)^{2}+3 e^{-2 \sigma}\left(\partial_{r} b\right)^{2}=2\left[F_{t r}^{2}+\frac{\left(F_{\varphi z}-C F_{t z}\right)^{2}}{D^{2}}\right] \\
& \left(\partial_{z} \phi\right)^{2}+e^{-2 \phi}\left(\partial_{z} a\right)^{2}+3\left(\partial_{z} \sigma\right)^{2}+3 e^{-2 \sigma}\left(\partial_{z} b\right)^{2}=4 \Omega^{2}-2\left[F_{t r}^{2}+\frac{\left(F_{\varphi z}-C F_{t z}\right)^{2}}{D^{2}}\right] .
\end{aligned}
$$

For all the solutions found above, the right-hand side of the above equations is a constant and, as a result, the dependence of the scalar fields on the coordinates $r$ and $z$ can be linearly at the most. When one considers the contribution of the electromagnetic field in 
the right-hand-side of eqs. (4.17)-(4.18), it turns out that these solutions form two distinct groups : for the Som-Raychaudhuri (4.7) and the Rebouças (4.8) ansatzes, the contribution is identically zero while, for the Som-Raychaudhuri-dual (4.9) and the Rebouças-dual (4.12) ansatzes, is not zero. This means that, for the first group, the scalar fields depend linearly only on the coordinate $z$ while, for the second group, a linear dependence also on the radial coordinate seems to be possible. However, when the corresponding expressions for the scalar fields are substituted into their equations of motion, the radial dependence disappears and the final solution takes the form

$$
\phi=\phi_{0}+\phi_{1} z \quad, \quad a=a_{0} \quad, \quad \sigma=\sigma_{0}+\sigma_{1} z \quad, \quad b=b_{0},
$$

where now

$$
\phi_{1}^{2}+3 \sigma_{1}^{2}=4 \Omega^{2} .
$$

The above solution strongly resembles the one derived in the neutral case. This result was to be expected due to the absence of any interaction between the electromagnetic and the scalar fields. We may then conclude that the only effect of the introduction of the electromagnetic field is the modification of the relation between the fundamental parameters of the theory : instead of $4 \Omega^{2}=m^{2}$ holding in the neutral case, we now have

$$
4 \Omega^{2}-m^{2}=Q^{2}
$$

where $Q$ plays the role of the electric or magnetic charge depending on the "flavour" of the component of the electromagnetic field considered each time. A result similar to (4.19) for a single scalar field minimally coupled to gravity and in the presence of an electromagnetic field was derived in [10]. Here, we presented the result for four scalar fields and for all possible configurations of the electromagnetic field that lead to a Gödel-type universe. Once again, the generalization of the above result for the case of multiple, minimally-coupled to the gravity, scalar fields is straightforward.

Finally, the question concerning the existence or not of Closed Timelike Curves in the presence of the electromagnetic field has to be addressed. The new relation (4.21) between the fundamental parameters of the theory leads to the result $4 \Omega^{2}>m^{2}$. When this inequality is put together with the expression (3.9), we may easily conclude that the appearance of Closed Timelike Curves for a certain range of values of the radial coordinate $r$ is inevitable. No matter how harmless the modification of the relation between the parameters of the theory may seem at first sight, it causes the breakdown of the causality in our Gödel-type axially-symmetric universe (3.8).

\section{$5 \quad$ EM : Non-minimal Coupling}

In the previous section, we considered all the possible configurations of an electromagnetic field that may lead to a charged Gödel-type universe. Although all of the solutions found are 
theoretically acceptable since they constitute solutions of the equations of motion, some of the ansatzes concerning the non-vanishing components of the electromagnetic field are more physically preferable. If we accept the assumption of a homogeneous distribution of electric charges over the universe, then, in the case of a Gödel-type universe, every point-charge rotating around the $z$-axis creates a current loop. The net effect of all these tiny currents is the creation of multiple "solenoids" around the $z$-axis with radii varying from zero to infinity. An observer located in a point along the $z$-axis would detect a constant magnetic field induced by the total current carrying by the "solenoids".

So, if we assume that $\vec{B}=\hat{z} \mu_{0} i$, where $i$ the total current and $\mu_{0}$ the permeability of the free space, and go back to constraints (4.1)-(4.3), we observe that, although the conditions (4.6) for a Gödel-type spacetime are still fulfilled, the constraint (4.2) is not satisfied in the case of the minimal coupling, i.e. in the case of a constant coupling function $f_{3}$. However, if we assume that the electromagnetic field is non-minimally coupled to gravity and assume the space-dependence of the coupling functions $f_{3}$ and $f_{4}$, the following relation

$$
4 \Omega^{2}-m^{2}=\mu_{0}^{2} i^{2}
$$

between the fundamental parameters is obtained once we assume that $f_{3}(r)=-D^{2}(r) / 4$. Note that, as in the minimal-coupling case, the coupling function $f_{3}$ has to be negative in order to ensure the positivity of the $t t$-component of the energy-momentum tensor of the electromagnetic field.

When the ansatz for the electromagnetic field is substituted into Maxwell's equations (A.27)-(A.30), we obtain the following expressions for the electric-charge density and the density of electric current along the $\hat{\varphi}$ direction

$$
\rho=\frac{\mu_{0} i}{4} \frac{(C D)^{\prime}}{D} \quad, \quad j^{\varphi}=-\frac{\mu_{0} i}{4} \frac{D^{\prime}}{D}
$$

respectively. While the density of the electric current vanishes on the $z$-axis and becomes constant at infinity as expected, the electric-charge density also vanishes at the origin but diverges at infinity. This ill behaviour of the electric-charge density makes our ansatz considerably less attractive. Moreover, a non-trivial source-term of the form $j^{t} A_{t}$ enters the Lagrangian putting in risk the Gödel-type character of our spacetime.

However, the consideration of a constant, magnetic field along the $z$-axis does not complete the picture of a charged, rotating universe. There are additional components of the electromagnetic field which are highly probable to exist and should be accommodated in our theory. For example, in a rotating universe, there might be a steady distribution of electric or magnetic monopoles along the $z$-axis, thus, creating a radial electric or magnetic field, respectively. We start with the introduction of a radial electric field : the conditions (4.6) for the existence of a Gödel-type universe are fulfilled and the fundamental relation (5.1) is recovered, if the electric field and the coupling function $f_{3}$ satisfy the following relations

$$
F_{t r}=\frac{F_{\varphi r}}{C(r)}=\frac{\mu_{0} i}{C^{2}(r)} \quad, \quad f_{3}=-\frac{C^{2}(r)}{4}
$$


respectively. The substitution of the combination of $F_{\varphi r}$ and $F_{t r}$ into Maxwell's equations (A.27)-(A.30) leads to the elimination of the charge-current density $j^{\varphi}$ while preserves the existence of the divergent electric-charge current $\rho$ and the corresponding source-term $j^{t} A_{t}$ in the Lagrangian.

As a last resort, we include in the theory a radial magnetic field $F_{z \varphi}$. By following a similar procedure, a Gödel-type universe does indeed arise if we assume, apart from the relations (5.3), that $F_{z \varphi}=D(r) F_{t r}$. When the three components $F_{\varphi r}, F_{t r}$ and $F_{z \varphi}$ are substituted into Maxwell's equations, we see that the above combination of the components of the electromagnetic field satisfies the source-free Maxwell's equations and that the illbehaved electric-charge density $\rho$ is eliminated once we assume that $f_{4}=f_{3}$. Actually, the above combination of electromagnetic components is a Rebouças-dual type of solution which in the minimal-coupling case satisfied the source-free equations as well. In the present case, we demanded a constant, magnetic field along the $z$-axis and, subsequently, a radial electric and magnetic field according to the expected distribution of charges and currents in a rotating universe. Then, the introduction of space-dependent coupling functions ensured the existence of a Gödel-type rotating universe.

Although the magnetic field takes on a constant value as we assumed motivated by the corresponding behaviour of a magnetic field in Minkowski spacetime, the other two components adopt a different behaviour. For a linear, constant distribution of electric or magnetic charges, in Minkowski spacetime, we anticipate to find a radial electric or magnetic field decreasing with the radial distance from the origin. However, in a Gödel-type spacetime, the electric field behaves as $E_{r} \sim 1 / r^{2}$ near the origin while decreases exponentially at infinity. On the other hand, the radial magnetic field scales as $B_{r} \sim 1 / r$ near the origin, as expected, but assumes a constant value at infinity which leads to an infinite total amount of magnetic charge. At the end of the day, the corresponding magnetic-charge density associated with $B_{r}$ cancels out the divergent electric-charge density $\rho$ leaving the three electromagnetic components to satisfy the source-free Maxwell's equations.

Finally, let us add here that the fundamental relation (5.1) leads again to $4 \Omega^{2}>m^{2}$ and to the existence of Closed Timelike Curves according to eq. (3.9). It seems that the introduction of an electromagnetic field, coupling minimally or non-minimally to gravity and being induced by an electric or magnetic charge or electric current, always results in the breakdown of causality in a Gödel-type universe. The accommodation of multiple, minimallycoupled to gravity, scalar fields of the form (4.19) although compatible with the above picture can not lead to the restoration of causality of our Gödel-type universe.

\section{$6 \quad$ EM and Scalar Fields : String Coupling}

In this section, we assume the presence of an electromagnetic and scalar fields minimallycoupled to gravity but with a non-trivial interaction between them. Thus, we assume that the coupling functions $f_{3}$ and $f_{4}$ are field-dependent (2.5) in agreement with the one-loop 
corrected superstring effective theory. For simplicity, we are going to consider the case where only the dilaton $\phi$ and axion $a$ fields are present in the theory and ignore the moduli fields $b$ and $\sigma$. More specifically, we consider the following action functional

$$
S_{e f f}=\int d^{4} x \sqrt{-g}\left\{\frac{R}{2}-\Lambda+\frac{1}{4}\left(\partial_{\mu} \phi\right)^{2}+\frac{1}{4} e^{-2 \phi}\left(\partial_{\mu} a\right)^{2}-\frac{\alpha^{\prime} e^{\phi}}{8 g^{2}} F^{\mu \nu} F_{\mu \nu}-\frac{\alpha^{\prime} a}{8 g^{2}} F \tilde{F}\right\}
$$

The possible configurations of the electromagnetic field capable of leading to Gödel-type cosmological solutions, in the minimal-coupling case, were studied in section 4 . Here, we will attempt to generalize those ansatzes in the case of field-dependent coupling functions in such a way as to ensure the existence of a Gödel-type universe. We anticipate that the dependence of the coupling functions on the scalar fields will be appropriately absorbed in the ansatzes for the electromagnetic components as well as in the expressions of the electric $j^{\mu}$ and magnetic $k^{\mu}$ four-currents. However, the coupling between the electromagnetic and scalar fields may result in a spacetime dependence for the fields $\phi$ and $a$ different from the one found in section 4. We may, thus, write the expressions for the scalar fields in the following way

$$
\phi(r, z)=\phi^{(0)}(z)+\alpha^{\prime} \tilde{\phi}(r, z) \quad, \quad a(r, z)=a^{(0)}+\alpha^{\prime} \tilde{a}(r, z)
$$

where $\phi^{(0)}$ and $a^{(0)}$ are the zero-order solution for the scalar fields which follow if we set $\alpha^{\prime}=0$ and which are given by eq. (3.17). Since, in the above Lagrangian, we have considered only terms up to $\mathcal{O}\left(\alpha^{\prime}\right)$ in the Regge slope, we must ignore all terms in the equations of motion that are of $\mathcal{O}\left(\alpha^{\prime 2}\right)$ or higher. As a result, the coupling functions $f_{3}$ and $f_{4}$ which are already of $\mathcal{O}\left(\alpha^{\prime}\right)$ will contain only the zero-order expression for the scalar fields and have the form

$$
f_{3}=-\frac{\alpha^{\prime} e^{\phi_{0}+\phi_{1} z}}{8 g^{2}} \quad, \quad f_{4}=-\frac{\alpha^{\prime} a_{0}}{8 g^{2}}
$$

We start with the Som-Raychaudhuri generalized ansatz for a magnetic field which can be written as

$$
F_{\varphi r}(r, z)=Q e^{-\phi / 2} D(r) .
$$

If we allow the above magnetic field to satisfy the generalized Maxwell's equations (4.10), we are led to the determination of the corresponding electric and magnetic-charge density necessary to support this magnetic field. They are given by

$$
\rho=\frac{\alpha^{\prime} \Omega}{4 g^{2}} Q e^{\phi / 2} \quad, \quad \eta=\frac{\alpha^{\prime} \phi_{1}}{16 g^{2}} Q e^{-\phi / 2},
$$

where, now, from eq. (3.18) $\phi_{1}^{2}=m^{2}$ and where we have set $a_{0}=1$ for simplicity. The more complex Rebouças ansatz now takes the form

$$
F_{t z}=Q e^{-\phi / 2} \sin (2 \Omega z) \quad, \quad F_{\varphi z}=C(r) F_{t z} \quad, \quad F_{\varphi r}=-Q e^{-\phi / 2} D(r) \cos (2 \Omega z)
$$


and the corresponding electric and magnetic-charge density are found to be

$$
\rho=\frac{\alpha^{\prime} \phi_{1}}{16 g^{2}} Q e^{\phi / 2} \sin (2 \Omega z) \quad, \quad \eta=-\frac{\alpha^{\prime} \phi_{1}}{16 g^{2}} Q e^{-\phi / 2} \cos (2 \Omega z) .
$$

The "dual" forms of the above ansatzes demand a rather complex distribution of electric and magnetic charges and currents. Namely, for the Som-Raychaudhuri-dual ansatz

$$
F_{z \varphi}=Q e^{-\phi / 2} D(r),
$$

we obtain

$$
\begin{array}{cc}
\rho=-\frac{\alpha^{\prime} \phi_{1}}{16 g^{2}} Q e^{\phi / 2} \frac{C}{D} \quad, & \eta=-\frac{\alpha^{\prime}}{8 g^{2}} Q e^{-\phi / 2} \frac{D^{\prime}}{D} \\
j^{\varphi}=\frac{\alpha^{\prime} \phi_{1}}{16 g^{2}} Q \frac{e^{\phi / 2}}{D} \quad, & k^{\varphi}=0,
\end{array}
$$

while, for the generalized Rebouças-dual ansatz

$$
F_{t r}=\frac{Q}{\sqrt{2}} e^{-\phi / 2} \quad, \quad F_{\varphi r}=C(r) F_{t r} \quad, \quad F_{\varphi z}=-D(r) F_{t r},
$$

we are led to the expressions

$$
\begin{array}{cc}
\rho=\frac{\alpha^{\prime} Q e^{\phi / 2}}{8 \sqrt{2} g^{2}}\left(\frac{D^{\prime}}{D}-\frac{\phi_{1} C}{2 D}\right) \quad, \quad \eta=-\frac{\alpha^{\prime} Q e^{-\phi / 2}}{8 \sqrt{2} g^{2}}\left(\frac{D^{\prime}}{D}-\frac{\phi_{1} C}{2 D}\right) \\
j^{\varphi}=\frac{\alpha^{\prime} \phi_{1} Q}{16 \sqrt{2} g^{2}} \frac{e^{\phi / 2}}{D} \quad, \quad k^{\varphi}=-\frac{\alpha^{\prime} \phi_{1} Q}{16 \sqrt{2} g^{2}} \frac{e^{-\phi / 2}}{D} .
\end{array}
$$

Finally, and in order to complete the picture, we reconsider the ansatzes (4.14) and (4.16), which in the minimal-coupling case were ruled out. In its appropriately generalized form, the ansatz (4.14) reads

$$
F_{t r}=Q e^{-\phi / 2} \quad, \quad F_{\varphi r}=C(r) F_{t r}
$$

leading to the results

$$
\begin{aligned}
\rho=\frac{\alpha^{\prime}}{8 g^{2}} Q e^{\phi / 2} \frac{D^{\prime}}{D} \quad, \quad \eta & =\frac{\alpha^{\prime} \phi_{1}}{16 g^{2}} Q e^{-\phi / 2} \frac{C}{D} \\
j^{\varphi}=0 \quad, \quad k^{\varphi} & =-\frac{\alpha^{\prime} \phi_{1}}{16 g^{2}} Q \frac{e^{-\phi / 2}}{D},
\end{aligned}
$$

while, for its "dual" ansatz

$$
F_{t z}=Q e^{-\phi / 2} \quad, \quad F_{\varphi z}=C(r) F_{t z},
$$


we obtain

$$
\rho=\frac{\alpha^{\prime} \phi_{1}}{16 g^{2}} Q e^{\phi / 2} \quad, \quad \eta=\frac{\alpha^{\prime} \Omega}{4 g^{2}} Q e^{-\phi / 2}
$$

In this form, with the electric and magnetic densities of charges and currents explicitly written, we may easily detect the actual electric-magnetic duality relations that hold between some of the ansatzes and connect $F_{\mu \nu}$ with $\tilde{F}^{\mu \nu}=\frac{1}{2} \eta^{\mu \nu \rho \sigma} F_{\rho \sigma}$. The Som-Raychaudhuri ansatz (6.4) and the ansatz (6.17) are connected by the duality transformations

$$
F \rightarrow-\tilde{F} \quad, \quad j^{\mu} \rightarrow k^{\mu} \quad, \quad k^{\mu} \rightarrow-j^{\mu} \quad, \quad \phi \rightarrow-\phi
$$

and the same holds for the Som-Raychaudhuri-dual ansatz (6.8) and the ansatz (6.14). The dilaton participates in the duality transformations due to the non-trivial coupling between this field and the electromagnetic one. The same duality but without the participation of the dilaton field was also valid in the minimal-coupling case studied in section 4 . This duality is always valid in the source-free case, breaks down when the electric four-current $j^{\mu}$ is included in the theory and is restored only after the introduction of the magnetic four-current $k^{\mu}$ [20].

All of the above generalized ansatzes for the electromagnetic field respect the conditions (4.6) and, in principle, lead to a Gödel-type rotating universe of the form (3.8). However, in almost all of the cases, a non-trivial source-term $\left(j^{\mu}+k^{\mu}\right) A_{\mu}$ enters the Lagrangian and subsequently the Einstein's equations destroying eventually the Gödel-type solution for the spacetime. In the case of the Som-Raychaudhuri-dual ansatz (6.8), we found the nonvanishing source-term $j^{\varphi} A_{\varphi}$ to be a constant. Such a source-term could be absorbed in the cosmological constant of the theory leading once again to a Gödel-type universe. However, it turns out that the combination of the source-term and the kinetic term $F_{\mu \nu} F^{\mu \nu}$ vanishes identically which leads us to the results of section 3 for an uncharged Gödel-type universe.

The only ansatz, for which this source-term is identically zero, is the generalized SomRaychaudhuri ansatz (6.4). As a result, this is the only acceptable solution for the electromagnetic field since it leaves unchanged the Einstein's equations while satisfies the sourcefull Maxwell's equations at the same time. On the other hand, the required distribution of electric and magnetic charges is finite and homogeneous in radial space and, thus, more physically favoured with the corresponding current densities being identically zero. For this solution, the constraint (4.2) leads to the following relation of the fundamental parameters of the theory

$$
4 \Omega^{2}-m^{2}=\frac{\alpha^{\prime}}{2 g^{2}} Q^{2} .
$$

We observe that the introduction of a non-trivial interaction between the scalar fields of the theory and the electromagnetic field does not restore the causality of the axially-symmetric spacetime since the above relation results, once again, in the appearance of Closed Timelike Curves.

Finally, we need to determine the solution for the scalar fields $\phi$ and $a$ in the presence of the $\alpha^{\prime}$-order electromagnetic field. For the only acceptable configuration, i.e. the Som- 
Raychaudhuri generalized ansatz (6.4) for a magnetic field along the $z$-axis, eqs. (A.23), (4.4) and (4.5) take the form

$$
\begin{aligned}
& \partial_{r} \phi \partial_{z} \phi+e^{-2 \phi} \partial_{r} a \partial_{z} a=0, \\
& \left(\partial_{r} \phi\right)^{2}+e^{-2 \phi}\left(\partial_{r} a\right)^{2}=0, \\
& \left(\partial_{z} \phi\right)^{2}+e^{-2 \phi}\left(\partial_{z} a\right)^{2}=4 \Omega^{2} .
\end{aligned}
$$

By taking into account the expressions (6.2), we conclude that each of the above terms involving derivatives of the axion field $a$ as well as the term $\left(\partial_{r} \phi\right)^{2}$ are of $\mathcal{O}\left(\alpha^{\prime 2}\right)$ and they should be ignored. Then, eq. (6.22) is trivially satisfied while eq. (6.21) leads once again to the independence of the dilaton field of the radial coordinate $r$. Eq. (6.23) reveals the fact that the dilaton field is linearly dependent on the coordinate $z$ leading to the result

$$
\phi(z)=\phi_{0}+\phi_{1} z\left(1+\frac{\alpha^{\prime} Q^{2}}{4 g^{2} m}\right) .
$$

Since the Einstein's equations can give us no information on the form of the axion field, we turn to the corresponding equation of motion (2.8). The combination $F \tilde{F}$ which acts as a source-term in the axion equation of motion is identically zero for the ansatz (6.4) and, thus, we obtain

$$
e^{-2\left(\phi_{0}+\phi_{1} z\right)} \frac{\partial}{\partial r}\left[D(r) \frac{\partial \tilde{a}(r, z)}{\partial r}\right]+D(r) \frac{\partial}{\partial z}\left[e^{-2\left(\phi_{0}+\phi_{1} z\right)} \frac{\partial \tilde{a}(r, z)}{\partial z}\right]=0 .
$$

By applying the method of separation of variables, the solution for the axion field takes the form

$$
a(r, z)=a_{0}+\alpha^{\prime} A_{0} \cosh (m r) \cos (m z),
$$

where $A_{0}$ is an arbitrary constant and where the reality of the axion field has been demanded.

It is worth noting that the existence of a Gödel-type rotating universe, in the presence of interacting scalar and electromagnetic fields, is incompatible with a universe empty from electric and magnetic charges and currents. For instance, if we demand that the SomRaychaudhuri ansatz (6.4) satisfies the source-free Maxwell's equations, we are led to the result $C=0$ and

$$
D(r)=\frac{1}{\mu} \sin (\mu r) \quad \text { with } \quad 2 \Lambda \equiv \mu^{2}=\frac{\alpha^{\prime}}{2 g^{2}} Q^{2} .
$$

Then, the line-element of the spacetime assumes the spherically-symmetric form

$$
d s^{2}=d t^{2}-d r^{2}-d z^{2}-\frac{\sin ^{2}(\mu r)}{\mu^{2}} d \varphi^{2}
$$

with the metric component $g_{\varphi \varphi}=-D^{2}(r)$ being always negative and, thus, excluding the presence of Closed Timelike Curves. Near the origin $D(r) \sim r$ and the above line-element 
resembles the Minkowski one. Finally, from eqs. (4.4) and (4.5) we find that the only acceptable solution for the scalar fields is $\phi=\phi_{0}=$ const. and $a=a_{0}=$ const. leading to their decoupling from the theory. The presence or not of the source terms in Maxwell's equations affects the form of the metric function $C(r)$ and thus the structure of the spacetime : while in the source-free case, the system of equations accepts only spherically-symmetric line-elements with no CTC's and a positive cosmological constant, in the source-full case, axially-symmetric Gödel-type solutions, that are characterized by a negative cosmological constant and the presence of CTC's, arise. Moreover, in the former case, the scalar fields are decoupled from the gravito-magnetic system, while, in the latter case, a first-order, in $\alpha^{\prime}$, solution for the scalar fields was found that completes the zero-order solution found in section 3 .

Let us, finally, note that, in the neutral case studied in section 3 , the negative sign of the cosmological constant was dictated by the relation $C^{\prime 2}=-2 \Lambda D^{2}$ and the reality of the metric functions. In all the charged cases, however, considered in this article, there was no constraint on the sign of $\Lambda$. Due to the fact that the original Gödel solutions were derived for a negative cosmological constant, we chose $\Lambda$ to have a negative sign in these cases, too. We would like to stress that Gödel-type solutions arise also for a positive cosmological constant. In this case, the conditions (4.6) take the form

$$
\frac{D^{\prime \prime}}{D}=\text { const. }=-2 \Lambda=-\mu^{2} \quad, \quad \frac{C^{\prime}}{D}=\text { const. }=2 \Omega
$$

and are still satisfied by all the configurations of the electromagnetic field considered in sections 4, 5 and 6 . However, the solution for the spacetime line-element, now, has the form

$$
d s^{2}=\left[d t+\frac{4 \Omega}{\mu^{2}} \sin ^{2}\left(\frac{\mu r}{2}\right) d \varphi\right]^{2}-d r^{2}-d z^{2}-\frac{\sin ^{2}(\mu r)}{\mu^{2}} d \varphi^{2}
$$

and the relations (4.21), (5.1) and (6.20) between the fundamental parameters of the theory are modified with $m^{2}$ being replaced by $-\mu^{2}$. The $g_{\varphi \varphi}$ component of the Gödel metric (6.30) is given by

$$
g_{\varphi \varphi}=-\frac{4}{\mu^{2}} \sin ^{2}\left(\frac{\mu r}{2}\right)\left[1-\left(1+\frac{4 \Omega^{2}}{\mu^{2}}\right) \sin ^{2}\left(\frac{\mu r}{2}\right)\right]
$$

and it is easy to see that the sign of the expression inside the brackets changes from positive to negative and vice versa in a harmonic way giving rise to successive causal and acausal regions in spacetime. This behaviour was originally found in the case of a, minimally-coupled to gravity, electromagnetic field [5] and, subsequently, in the framework of other theories as well $[18$.

\section{Conclusions}

In this paper, we have addressed the question of the existence of axially-symmetric Gödeltype solutions in the framework of gravitational theories formulated in a way similar to 
the one-loop superstring effective theory. The incorporation of minimally-coupled to gravity scalar fields as well as a non-vanishing electromagnetic field, either minimally or nonminimally coupled to gravity, was proved to be consistent with the concept of Gödel-type rotating universes. The coupling functions of the electromagnetic field were assumed to be zero, constant, space-dependent or field-dependent with various consequences each time on the structure of spacetime and the solution for the scalar fields.

In the absence of an electromagnetic field, the system of the four scalar fields of the superstring effective theory, all minimally-coupled to gravity, was studied in an axiallysymmetric background. Assuming also a vanishing cosmological constant, the above system inevitably leads to a spherically-symmetric spacetime background and to the decoupling of the scalar fields from the theory. Once a non-vanishing, negative value for the cosmological constant is assumed, an axially-symmetric solution for the spacetime of a Gödel type does arise with the scalar fields either depending linearly on the $z$ coordinate or adopting a constant value. The resulting solution for the spacetime is an "extreme" Gödel-type universe with the fundamental parameters of the theory satisfying the relation : $4 \Omega^{2}=m^{2}$. It is exactly this relation that ensures the non-existence of Closed Timelike Curves and the nonviolation of causality in our Gödel-type universe. However, as it is known in the literature, this spacetime solution is "on the verge" of displaying the breakdown of causality which is realized when $4 \Omega^{2}$ becomes even slightly larger that $m^{2}$. The introduction of multiple, minimally-coupled to gravity, scalar fields is straightforward leaving the solution for the spacetime unchanged.

Next, we studied the charged case, and the corresponding equations of motion were rewritten in a convenient way which allowed us to determine first the solution for the metric functions in terms of the electromagnetic ansatz considered each time and subsequently the solution for the scalar fields. We started with the case of minimally-coupled electromagnetic and scalar fields assuming constant values for the two coupling functions $f_{3}$ and $f_{4}$. The gravito-electromagnetic system was examined thoroughly, in the presence of a negative cosmological constant, and apart from the two solutions already known in the literature, the Som-Raychaudhuri solution and the Rebouças solution, two new configurations were determined. They follow from the aforementioned solutions once one interchanges the indices $r$ and $z$ in the non-vanishing components of the electromagnetic field. The invariance of the equations of motion under this interchange results in the fulfillment of the Gödel conditions by these new ansatzes as well. As a result, a Gödel-type rotating universe emerges but, now, the relation between the fundamental parameters of the theory has become $4 \Omega^{2}>m^{2}$. This means that Closed Timelike Curves appear for a certain range of the radial coordinate and the causality is violated. The introduction of the electromagnetic field has evidently destroyed the fragile balance between the violation and the conservation of causality that characterized the neutral case. On the other hand, the scalar fields assume a form similar to the one found in the neutral case due to the absence of any interaction with the electromagnetic field.

The more physically favoured ansatz of a constant magnetic field along the rotation axis 
and a radial electric and magnetic component was studied next. This specific ansatz follows from the anticipated distribution of magnetic and electric charges and currents in a rotating universe : point-charges that create current loops around the rotation axis and a static distribution of electric and magnetic monopoles along the same axis. For such a configuration, the Gödel conditions are again fulfilled and the Maxwell's equations are satisfied as long as we assume that both of the coupling functions $f_{3}$ and $f_{4}$ have the same dependence on the radial coordinate. Then, a Gödel-type solution for the spacetime does arise which is again characterized by the violation of causality and the appearance of Closed Timelike Curves. The incorporation of minimally-coupled scalar fields although straightforward and compatible with the above results can not restore the causality in the universe.

Finally, the coupling functions $f_{3}$ and $f_{4}$ were assumed to have the field-dependent form of the superstring effective theory. In this case, there is a non-trivial coupling between the scalar fields of the theory and the electromagnetic field. The possible configurations of the electromagnetic field that could lead to a Gödel-type rotating universe were re-examined. Several such configurations were found but only one of them, the generalized Som-Raychaudhuri ansatz of a magnetic field along the rotation axis, managed to satisfy the Gödel conditions and the corresponding source-full Maxwell's equations at the same time. Once again, the relation $4 \Omega^{2}>m^{2}$, that holds in this case too, leads to the appearance of Closed Timelike Curves. The non-trivial coupling between the scalar fields and the electromagnetic field, although not able to restore the causality, leads to different results for the scalar fields and especially for the axion field $a$. While the dilaton field depends only linearly on the $z$ coordinate as in the previous cases, the axion field abandons the constant value adopted so far and assumes a form which strongly depends on both $r$ and $z$ coordinates.

In conclusion, a charged, string-inspired, gravitational theory of scalar and electromagnetic fields of the form considered in this article always accepts axially-symmetric Gödel-type solutions in the presence of a non-vanishing, negative cosmological constant. Gödel-type solutions arise also in the charged case with a positive cosmological constant, however, they are shown to lead to successive causal and acausal regions in spacetime. Only in the neutral case, where we ignore the electromagnetic field, we are able to ensure the non-violation of causality in the universe in agreement with previous results by Barrow and Dabrowski [18]. Once the electromagnetic field is introduced, the emergence of the Gödel-type solution is always accompanied by the appearance of Closed Timelike Curves and the violation of causality. However, the realization of the causally-ill Gödel solutions in the universe should be impeded by physical reasons. The uniformity of the Cosmic Microwave Background Radiation, as dictated by the COBE data, leads to the conclusion that the rate of the rigid rotation of our universe, if existent at all, should be extremely small. On the other hand, there is only weak indication for a global charge in the universe with the bulk of astrophysical objects being neutral. As a result, it is highly improbable that our universe would adopt the geometry of a Gödel cosmological solution, thus, excluding the possibility of the appearance of Closed Timelike Curves and the violation of causality. Finally, an interesting question that still remains open is what is the effect of the higher-order perturbative terms in $\alpha^{\prime}$ on the causal pathologies of the Gödel-type solutions derived in the framework of the superstring effective 
action. It is rather plausible that the presence of these terms, even if it is not capable of resolving the appearance of CTC's, would render the stringy Gödel-type solutions unstable, thus, depriving them from any physical significance.

Acknowledgments P.K. would like to thank Marcelo J. Rebouças for enlightening discussions. P.K. would also like to acknowledge financial support from the research program חENE $\Delta-95$ of the Greek Ministry of Science and Technology and from DOE grant DEFG02-94ER40823 at Minnesota during the early and late stages, respectively, of this work.

\section{Appendix: Useful formulae and equations}

For the axially-symmetric ansatz (2.6) for the metric tensor of the universe, we find the following results for the non-vanishing components of the fully-covariant curvature tensor $R_{\mu \nu \rho \sigma}$

$$
\begin{aligned}
& R_{t r t r}=\frac{C^{\prime 2}}{4 D^{2}}, \quad R_{t r t \varphi}=\frac{C C^{\prime 2}}{4 D^{2}}, \\
& R_{t r r \varphi}=-\frac{C^{\prime \prime}}{2}-\frac{C C^{\prime 2}}{4 D^{2}}+\frac{C^{\prime} D^{\prime}}{2 D} \quad, \quad R_{t \varphi t \varphi}=\frac{C^{\prime 2}}{4} \\
& R_{r \varphi r \varphi}=C C^{\prime \prime}-D D^{\prime \prime}+\frac{3}{4} C^{\prime 2}+\frac{C C^{\prime 2}}{4 D^{2}}-\frac{C C^{\prime} D^{\prime}}{D}
\end{aligned}
$$

the Ricci tensor $R_{\mu \nu}$

$$
\begin{aligned}
& R_{t t}=-\frac{C^{\prime 2}}{2 D^{2}} \quad, \quad R_{r r}=\frac{D^{\prime \prime}}{D}-\frac{C^{2}}{2 D^{2}} \\
& R_{\varphi \varphi}=-C C^{\prime \prime}+D D^{\prime \prime}-\frac{C^{\prime 2}}{2}-\frac{C^{2} C^{\prime 2}}{2 D^{2}}+\frac{C C^{\prime} D^{\prime}}{D} \\
& R_{t \varphi}=-\frac{C^{\prime \prime}}{2}-\frac{C^{\prime}}{2 D^{2}}\left(C C^{\prime}-D D^{\prime}\right)
\end{aligned}
$$

and the scalar curvature $R$

$$
R=-\frac{2 D^{\prime \prime}}{D}+\frac{C^{\prime 2}}{2 D^{2}}
$$

respectively. By using the above expressions, the non-vanishing components of the Einstein tensor $G_{\mu \nu}$ are the following

$$
G_{t t}=-\frac{3}{4} \frac{C^{\prime 2}}{D^{2}}+\frac{D^{\prime \prime}}{D} \quad, \quad G_{r r}=-\frac{C^{\prime 2}}{4 D^{2}}
$$




$$
\begin{aligned}
& G_{\varphi \varphi}=-C C^{\prime \prime}+\frac{C^{2} D^{\prime \prime}}{D}-\frac{C^{2}}{4}-\frac{3 C^{2} C^{2}}{4 D^{2}}+\frac{C C^{\prime} D^{\prime}}{D} \\
& G_{z z}=\frac{C^{\prime 2}}{4 D^{2}}-\frac{D^{\prime \prime}}{D} \\
& G_{t \varphi}=-\frac{C^{\prime \prime}}{2}+\frac{C D^{\prime \prime}}{D}-\frac{3 C C^{2}}{4 D^{2}}+\frac{C^{\prime} D^{\prime}}{2 D}
\end{aligned}
$$

If we assume that, due to the symmetry of the line-element of the spacetime, the scalar fields depend only on $r$ and $z$, the corresponding components of the energy-momentum tensor $T_{\mu \nu}^{s}$ take the form

$$
\begin{aligned}
& T_{t t}^{s}= \frac{1}{4}\left\{\left(\partial_{r} \phi\right)^{2}+\left(\partial_{z} \phi\right)^{2}+e^{-2 \phi}\left[\left(\partial_{r} a\right)^{2}+\left(\partial_{z} a\right)^{2}\right]+3\left[\left(\partial_{r} \sigma\right)^{2}+\left(\partial_{z} \sigma\right)^{2}\right]+\right. \\
&\left.3 e^{-2 \sigma}\left[\left(\partial_{r} b\right)^{2}+\left(\partial_{z} b\right)^{2}\right]\right\} \\
& T_{r z}^{s}= \frac{1}{2}\left\{\partial_{r} \phi \partial_{z} \phi+e^{-2 \phi} \partial_{r} a \partial_{z} a+3 \partial_{r} \sigma \partial_{z} \sigma+3 e^{-2 \sigma} \partial_{r} b \partial_{z} b\right\} \\
& T_{r r}^{s}=\frac{1}{4}\left\{\left(\partial_{r} \phi\right)^{2}-\left(\partial_{z} \phi\right)^{2}+e^{-2 \phi}\left[\left(\partial_{r} a\right)^{2}-\left(\partial_{z} a\right)^{2}\right]+3\left[\left(\partial_{r} \sigma\right)^{2}-\left(\partial_{z} \sigma\right)^{2}\right]+\right. \\
&\left.\quad 3 e^{-2 \sigma}\left[\left(\partial_{r} b\right)^{2}-\left(\partial_{z} b\right)^{2}\right]\right\}, \\
& T_{z z}^{s}=-T_{r r}^{s}, \quad T_{\varphi \varphi}^{s}=\left(C^{2}-D^{2}\right) T_{t t}^{s} \quad, \quad T_{t \varphi}^{s}=C T_{t t}^{s} .
\end{aligned}
$$

In the presence of a non-vanishing electromagnetic field, the Einstein's equations take the form

$$
\begin{aligned}
& -\frac{3}{4} \frac{C^{\prime 2}}{D^{2}}+\frac{D^{\prime \prime}}{D}+\Lambda+4 f_{3}\left(g^{r r} F_{t r}^{2}+g^{z z} F_{t z}^{2}+g^{\varphi \varphi} F_{t \varphi}^{2}-\frac{F^{2}}{4}\right)=-T_{t t}^{s} \\
& -\frac{C^{\prime 2}}{4 D^{2}}-\Lambda+4 f_{3}\left(g^{t t} F_{t r}^{2}+g^{z z} F_{r z}^{2}+g^{\varphi \varphi} F_{r \varphi}^{2}+2 g^{t \varphi} F_{t r} F_{\varphi r}+\frac{F^{2}}{4}\right)=-T_{r r}^{s}, \\
& \frac{C^{\prime 2}}{4 D^{2}}-\frac{D^{\prime \prime}}{D}-\Lambda+4 f_{3}\left(g^{t t} F_{z t}^{2}+g^{r r} F_{z r}^{2}+g^{\varphi \varphi} F_{z \varphi}^{2}+2 g^{t \varphi} F_{z t} F_{z \varphi}+\frac{F^{2}}{4}\right)=-T_{z z}^{s} \\
& -C C^{\prime \prime}+\frac{C^{2} D^{\prime \prime}}{D}-\frac{C^{\prime 2}}{4}-\frac{3 C^{2} C^{2}}{4 D^{2}}+\frac{C C^{\prime} D^{\prime}}{D}+\Lambda\left(C^{2}-D^{2}\right)
\end{aligned}
$$




$$
\begin{gathered}
+4 f_{3}\left[g^{t t} F_{\varphi t}^{2}+g^{r r} F_{\varphi r}^{2}+g^{z z} F_{\varphi z}^{2}-\frac{F^{2}}{4}\left(C^{2}-D^{2}\right)\right]=-T_{\varphi \varphi}^{s}, \\
-\frac{C^{\prime \prime}}{2}+\frac{C D^{\prime \prime}}{D}-\frac{3 C C^{\prime 2}}{4 D^{2}}+\frac{C^{\prime} D^{\prime}}{2 D}+\Lambda C \\
+4 f_{3}\left(g^{r r} F_{t r} F_{\varphi r}+g^{z z} F_{t z} F_{\varphi z}-g^{t \varphi} F_{t \varphi}^{2}-\frac{F^{2}}{4} C\right)=-T_{\varphi t}^{s}, \\
g^{z z} F_{t z} F_{r z}+g^{\varphi \varphi} F_{t \varphi} F_{r \varphi}+g^{t \varphi} F_{t \varphi} F_{r t}=0, \\
g^{r r} F_{t r} F_{z r}+g^{\varphi \varphi} F_{t \varphi} F_{z \varphi}+g^{t \varphi} F_{t \varphi} F_{z t}=0, \\
4 f_{3}\left[g^{t t} F_{r t} F_{z t}+g^{\varphi \varphi} F_{r \varphi} F_{z \varphi}+g^{t \varphi}\left(F_{r t} F_{z \varphi}+F_{z t} F_{r \varphi}\right)\right]=-T_{r z}^{s}, \\
g^{t t} F_{r t} F_{\varphi t}+g^{z z} F_{r z} F_{\varphi z}+g^{t \varphi} F_{r \varphi} F_{\varphi t}=0, \\
g^{t t} F_{z t} F_{\varphi t}+g^{r r} F_{z r} F_{\varphi r}+g^{t \varphi} F_{z \varphi} F_{\varphi t}=0,
\end{gathered}
$$

where $F^{2}=F^{\mu \nu} F_{\mu \nu}$ is given by

$$
\begin{gathered}
F^{2}=2\left\{g^{t t}\left(-F_{t r}^{2}-F_{t z}^{2}+g^{\varphi \varphi} F_{t \varphi}^{2}\right)+F_{r z}^{2}-g^{\varphi \varphi}\left(F_{r \varphi}^{2}+F_{z \varphi}^{2}\right)-\right. \\
\left.\left(g^{t \varphi} F_{t \varphi}\right)^{2}-2 g^{t \varphi}\left(F_{t r} F_{\varphi r}+F_{t z} F_{\varphi z}\right)\right\}
\end{gathered}
$$

and $T_{\mu \nu}^{s}$ denotes the energy-momentum tensor of the scalar fields given above.

In the charged case, the components of Einstein's equations are supplemented by a set of four additional equations, the Maxwell's equations, that come from the equation of motion of the electromagnetic field (2.12) and have the form

$$
\begin{aligned}
& \partial_{r}\left\{\frac{f_{3}}{D}\left[\left(D^{2}-C^{2}\right) F_{r t}+C F_{r \varphi}\right]\right\}+\partial_{r}\left(f_{4} F_{z \varphi}\right) \\
& +\frac{1}{D} \partial_{z}\left\{f_{3}\left[\left(D^{2}-C^{2}\right) F_{z t}+C F_{z \varphi}\right]\right\}-\partial_{z}\left(f_{4} F_{r \varphi}\right)=0, \\
& D \partial_{z}\left(f_{3} F_{z r}\right)-\partial_{z}\left(f_{4} F_{t \varphi}\right)=0,
\end{aligned}
$$




$$
\begin{aligned}
& \partial_{r}\left(D f_{3} F_{r z}\right)+\partial_{r}\left(f_{4} F_{t \varphi}\right)=0, \\
& \partial_{r}\left\{\frac{f_{3}}{D}\left(F_{r \varphi}-C F_{r t}\right)\right\}+\frac{1}{D} \partial_{z}\left\{f_{3}\left(F_{z \varphi}-C F_{z t}\right)\right\} \\
&-\partial_{r}\left(f_{4} F_{t z}\right)+\partial_{z}\left(f_{4} F_{t r}\right)=0 .
\end{aligned}
$$

As a result, any solution for the metric functions $C(r)$ and $D(r)$ and the various components of the electromagnetic field has to satisfy also the above four equations.

\section{References}

[1] K. Gödel, Rev. Mod. Phys. 21 (1949) 447.

[2] S.W. Hawking and G.F.R. Ellis, The Large Scale Structure of Space-time, Cambridge University Press, Cambridge (1973).

[3] H. Weyl, Space, Time and Matter, transl. H. Brose, Methuen, London (1921) ;

P. Nahin, Time Machines, Springer-Verlag (1993);

K.S. Thorne, Black Holes and Time Warps : Einstein's Outrageous Legacy, Picador, London (1994);

J.D. Barrow, Impossibility: the science of limits and the limits of science, Oxford University Press, Oxford (1998).

[4] M.M. Som and A.K. Raychaudhuri, Proc. R. Soc. London A 304 (1968) 81.

[5] M.J. Rebouças, Phys. Lett. A 70 (1979) 161.

[6] A.K. Raychaudhuri and S.N. Guha Thakurta, Phys. Rev. D 22 (1980) 802.

[7] W.A. Hiscock, Phys. Rev. D 17 (1978) 1497 ;

S.K. Chakraborty and N. Bandyopadhyay, J. Math. Phys. 24 (1983) 129 ;

V.F. Panov, Sov. Phys. J. 34 (1991) 130.

[8] V.M. Villalba, Mod. Phys. Lett. A 8 (1993) 3011 ;

L.O. Pimentel, A. Camacho and A. Macias, Mod. Phys. Lett. A 9 (1994) 3703.

[9] V.G. Krechet and I.V. Sandina, Sov. Phys. J. 24 (1982) 950.

[10] M.J. Rebouças and J. Tiomno, Phys. Rev. D 28 (1983) 1251.

[11] D.A. Leahy, Int. J. Theor. Phys. 21 (1982) 703 ;

A. Herrera and G.N. Shikin, Grav. Cosmol. 2 (1996) 155. 
[12] M.J. Rebouças, J.E. Aman and A.F.F. Teixeira, J. Math. Phys. 27 (1986) 1370.

[13] A.J. Accioly and A.T. Goncalves, J. Math. Phys. 28 (1987) 1547 ;

A.J. Accioly, Nuovo Cim. 100 B (1987) 703 ;

A.J. Accioly and G.E.A. Matsas, Phys. Rev. D 38 (1988) 1083.

[14] W.B. Bonnor, N.O. Santos and M.A.H. MacCallum, Class. Quant. Grav. 15 (1998) 357.

[15] M. Rooman and P. Spindel, Class. Quant. Grav. 15 (1998) 3241.

[16] M.J. Rebouças and A.F.F. Teixeira, J. Math. Phys. 39 (1998) 2180 ; Int. J. Mod. Phys. A 13 (1998) 3181;

H.L. Carrion, M.J. Rebouças and A.F.F. Teixeira, gr-qc/9904074.

[17] J.E. Aman, J.B. Fonseca-Neto, M.A.H. MacCallum and M.J. Rebouças, Class. Quant. Grav. 15 (1998) 1089 ;

J.B. Fonseca-Neto and M.J. Rebouças, Gen. Rel. Grav. 30 (1998) 1301.

[18] J.D. Barrow and M.P. Dabrowski, Phys. Rev. D 58 (1998) 103502.

[19] M.O. Calvão, M.J. Rebouças, A.F.F. Teixeira and W.M. Silva, Jr., J. Math. Phys. 29 (1988) 1127.

[20] P.A.M. Dirac, Phys. Rev. 74 (1948) 817 ;

L. Alvarez-Gaume and S.F. Hassan, Fortsch. Phys. 45 (1997) 159. 